\title{
Recomendaciones nacionales para el estudio y tratamiento del cáncer de pulmón durante la contingencia causada por COVID-19 en Colombia
}

\author{
National recommendations for the study \\ and treatment of lung cancer during \\ contingency caused by COVID-19 in Colombia
}

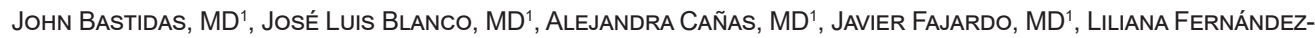
Trujillo, MD ${ }^{1}$, Plutarco García-Herreros, MD¹, Natalia Londoño, MD¹, Carlos Matiz, MD ${ }^{1}$, Jovani Osorno, MD¹, Nelson PÁez, MD ${ }^{1}$, Isabel Palacio, MD ${ }^{1}$, Audrey Piotrostanalzki, MD ${ }^{1}$, LuCia Viola, MD ${ }^{1}$, Franco Montufar, MD' ${ }^{1}$, Álvaro Casallas, MD², Stella Martínez, MD², DarWin Padilla, MD², Adriana Serna, MD²,

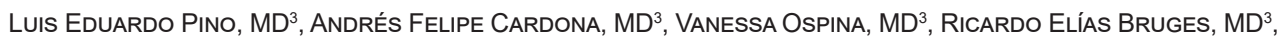

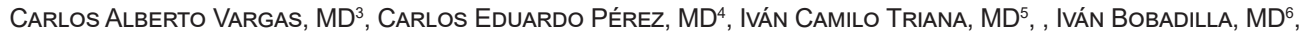
ILSE VÁSQuez, MD ${ }^{6}$, RicARdo Cendales, MD ${ }^{6}$.
\end{abstract}

\section{Introducción}

El termino cáncer comprende un grupo de enfermedades que comparten la característica de crecimiento no controlado de células anormales en un órgano o tejido determinado, que lleva a invasión de los tejidos adyacentes o diseminación a distancia a otros órganos o tejidos. Es la segunda causa de muerte a nivel mundial, con un estimado de 9,6 millones en el año 2018 (1).

Según datos de la Organización Mundial de la Salud (OMS), en el año 2018, el cáncer de pulmón representó el $11,6 \%$ de la totalidad de la enfermedad por cáncer en el mundo, con una mortalidad del 18,4\%. En Suramérica se reportaron 68504 casos, de los cuales, 29249 correspondían a nuevos casos; la mortalidad reportada fue de 62922 casos (2). Esto nos lleva a plantearnos la necesidad de realizar un diagnóstico y tratamiento oportuno del cáncer pulmonar.

En diciembre de 2019 se describieron los primeros casos de neumonía generada por un nuevo coronavirus de origen animal: el coronavirus 2 del síndrome respiratorio agudo severo (SARS-CoV-2), que genera la enfermedad denominada COVID-19 (3).

La OMS catalogó a la enfermedad por COVID-19 como una emergencia de salud pública de importancia internacional. En Colombia se confirmó el primer caso el 6 de marzo de 2020. Es una prioridad comunicarse con los pacientes, explicarles la situación actual a nivel mundial y local, y dar el soporte requerido para mantener un buen estado físico, mental y emocional durante la crisis, en busca del mejor plan terapéutico para cada caso (4).

Los pacientes con cáncer de pulmón tienen más probabilidad de infectarse con el SARS-CoV-2, en comparación con la población general. Entre los pacientes infectados con cáncer, el cáncer de pulmón es el más común, por lo que es necesario suministrar recomendaciones de tratamiento individualizado a estos pacientes. Consideramos importante generar medidas de prevención contra el coronavirus, ya que los hospitales se han convertido en áreas de alto riesgo de infección en pa-
${ }^{1}$ Médico especialista en Medicina Interna y Neumología; Miembro del Comité de Cáncer de la Asociación Colombiana de Neumología y Cirugía de Tórax. ${ }^{2}$ Médico especialista en Cirugía General y Cirugía de Tórax; Miembro del Comité de Cáncer de la Asociación Colombiana de Neumología y Cirugía de Tórax.

${ }^{3}$ Médico especialistas en Hematología y Oncología; Asociación Colombiana de Hematología y Oncología.

${ }^{4}$ Coautor asociado; Médico especialista en Infectología, Clínica de Marly, Bogotá. ${ }^{5}$ Coautor asociado; Médico rural de Investigación, Fundación Santa Fe de Bogotá, Instituto de Cáncer Carlos Ardila Lülle.

${ }^{6}$ Médico especialista en Radioterapia Oncológica; Asociación Colombiana de Radioterapia Oncológica.

Autor de correspondencia:

John Bastidas.

Correo electrónico:

jebastidas@hotmail.com

Recibido: 01/05/2020

Aceptado: 25/05/2020 
cientes oncológicos, quienes pueden tener coexistencias de factores de riesgo adicionales, como edad avanzada, estado de inmunocompromiso, desnutrición, alteración en la función o en la estructuralidad pulmonar, lo que genera mayor susceptibilidad a la infección por SARSCov-2, con riesgo de convertirse fácilmente en pacientes graves y críticamente enfermos.

Un estudio publicado en la revista Lancet Oncology confirmó que entre los pacientes infectados con SARS-CoV-2, aquellos con tumores malignos tuvieron un promedio de 13 días más de enfermedad grave, con deterioro significativamente más rápido, en comparación con los pacientes con tumores no malignos. El deterioro de los pacientes con tumor fue significativamente más rápido, y la tasa de enfermedad grave es aproximadamente 5 veces mayor que la de los pacientes sin tumores malignos $(39 \%$ y $8 \%$, respectivamente, $P=0,0003)(5,6)$.

\section{Recomendaciones para el diagnóstico y la estadificación del cáncer durante la pandemia por COVID-19}

En tiempo de pandemia, el cáncer pulmonar impone varios retos fundamentales en su diagnóstico. El primero es el relacionado con los síntomas, que en pacientes con neoplasia avanzada de consulta tardía podrían confundirse con los asociados con la neumonía por el nuevo coronavirus, el cual presenta dificultad respiratoria y hemoptisis; el segundo reto es que los pacientes con neoplasia pulmonar no confirmada puedan estar simultáneamente infectados por COVID-19; finalmente, el tercer reto, se genera por el cierre de la mayoría de los servicios ambulatorios; es posible que no se estén llevando a cabo los procedimientos requeridos para un diagnóstico temprano y estadificación en cáncer pulmonar.

Basados en datos de otros países en fases más avanzadas de la pandemia, y sus diferentes estrategias para los pacientes con cáncer, nos permitimos emitir algunas recomendaciones para hospitales con servicios de oncología o institutos de cáncer, en el escenario del COVID-19 y sospecha de cáncer pulmonar. Estas recomendaciones se deben evaluar en el contexto temporal de la pandemia, las características propias del centro en donde se preste la atención del paciente, y las políticas locales generadas en torno a la pandemia.
- Dado que no siempre se cuenta con la información epidemiológica de los pacientes, en caso de sospecha de cáncer pulmonar, considerarlos a todos como potencialmente infectados por SARS-CoV-2, $\mathrm{y}$ usar todos los elementos de protección personal (EPP) pertinentes.

- Realizar un triaje de los síntomas respiratorios, para reducir la exposición al COVID-19 del personal de salud y otros pacientes presentes en las instituciones de cuidado oncológico (7).

- Los pacientes con cáncer de pulmón en etapa de tratamiento o en diagnóstico y estadificación, que presenten síntomas respiratorios agudos o fiebre, se deben evaluar según la ruta propia de pacientes con sospecha de infección por SARS-CoV-2 de forma temprana (8).

- Disminuir la frecuencia de visitas a los hospitales y centros de oncología, a través de estrategias, como la teleconsulta, previa autorización del paciente y dejando registro en la historia clínica. Considerar la consulta presencial exclusivamente para los pacientes nuevos sin síntomas o con hallazgos sugestivos de COVID-19, quienes luego deben realizar seguimiento por teleconsulta (9).

- Implementar una plataforma de teleconsulta y teleseguimiento, con posibilidad de realizar actividades de reformulación, si es el caso, y vincularlo con una gestión administrativa de autorizaciones y programación completamente virtual.

- Debe solicitarse a los aseguradores facilidades para la gestión administrativa de autorizaciones de paraclínicos y entrega de medicamentos.

- Las juntas de tumores y reuniones académicas deben realizarse en forma virtual y como apoyo en casos de conducta controversial asociada con la pandemia o en aquellos seleccionados.

- Se consideran pacientes con alta prioridad para solicitud de estudios imagenológicos aquellos con síntomas respiratorios relevantes, que se consideren asociados con cáncer o con tratamiento; estadificación en sospecha de cáncer no estadificado, biopsias de nódulos sospechosos, evaluación de res- 
puesta de tratamiento en los primeros 6 meses o en cualquier momento, si se sospecha progresión. En estos pacientes se propone tomografía axial computada (TAC) de tórax (10).

- Procurar los procedimientos diagnósticos que generen la menor cantidad de aerosoles posible. Preferir, si se puede, la punción guiada por sobre la broncoscopia, siempre que sea técnicamente posible. Si la mejor técnica diagnóstica es la broncoscopia:

- Realizar interrogatorio sobre posibles síntomas o contacto con personas con COVID-19.

- En caso de tratarse de un caso con COVID-19 con lesión sugestiva de neoplasia, ajustarse a los tiempos de negativización de las pruebas y estabilidad clínica de los pacientes. Realizar procedimientos 4 semanas después de diagnóstico o 2 semanas luego de la negativización de la prueba.

- Considerar la actual fase de mitigación de la enfermedad y, por tanto, portar todo el equipo de protección personal, como potencial caso COVID-19.

- Realizar los estudios de estadificación pulmonar requeridos, ello incluye la endosonografía bronquial, especialmente cuando las infecciones respiratorias, que incluyen el COVID-19, pudieren generar adenomegalias que generen confusión y descarten un procedimiento quirúrgico en un paciente potencialmente candidato a cirugía. En todos los casos se debe usar el EPP completo y se deben seguir las recomendaciones para broncoscopia antes mencionadas.

- En los centros oncológicos, los pacientes en tratamiento sistémico deben asistir con un solo acompañante, usando tapabocas convencional. El equipo de salud que tiene contacto con aerosoles debe utilizar respiradores N95 o N100, con máscara elastómerica, monogafas, protector o visor facial, bata antifluido y guantes. Se debe realizar limpieza estricta de los equipos, que incluyen celulares, y de los muebles y la infraestructura en general. Realizar el lavado de manos, según las recomendaciones de la OMS, al iniciar y finalizar la visita.

- Considerar la utilización de recursos tecnológicos para el seguimiento de los pacientes en cuarente- na, por ejemplo, implementación de aplicaciones a través de plataformas Android o iOS, las cuales ya se han usado en otros países como herramientas de comunicación y educación.

Los casos considerados como verdaderas emergencias en pacientes oncológicos, independiente de su estado de infección, y que, por tanto, deben realizarse en menos de 48 horas, son:

- Atelectasia masiva pulmonar

- Obstrucción de vía aérea central traqueal o bronquial

- Hemoptisis masiva

- Migración de endoprótesis bronquial o traqueal.

En todos los casos que se realice procedimiento diagnóstico o terapéutico de emergencia se deben tener todas las medidas de protección personal, presumiendo que el paciente puede estar potencialmente infectado por SARS-CoV-2.

\section{Consideraciones quirúrgicas en el estudio y tratamiento del paciente con cáncer pulmonar durante la pandemia por COVID-19}

Para plantear las recomendaciones a este respecto es necesario revisar los análisis provenientes de China, de donde obtenemos, a la fecha, la evidencia más robusta disponible, teniendo en cuenta la velocidad de la pandemia. Según una publicación en The Lancet Oncology del 12 de febrero, en ese momento había 42747 casos confirmados, 1017 muertes y ya se reportaban casos en 25 países (6). Los pacientes con cáncer son más susceptibles a la infección debido a su estado de inmunosupresión sistémica y a los tratamientos oncológicos, como quimioterapia, radioterapia o cirugía. En los 2007 casos positivos analizados, el 18\% tenía diagnóstico de cáncer, siendo más frecuente el de pulmón, en $28 \%$ de los caso. Estos pacientes oncológicos también eran los de mayor edad, comúnmente asociados con historia de tabaquismo, que tuvieron más polipnea y su escenografía de base tenía peores hallazgos. Pero, lo más relevante de la descripción de los casos de COVID-19 asociados con cáncer en China es que tuvieron mayor necesidad de ventilación mecánica o murieron más. En este contexto se hace necesario evaluar cada 
caso por separado y definir si el riesgo de contagio es más o menos determinante que la indicación del procedimiento (11).

Hasta el momento, la literatura disponible respalda el concepto de reducir al mínimo la cirugía electiva, excepto aquellas para cáncer en determinadas condiciones. Para ayudar en la toma de decisiones al respecto es necesario precisar algunos aspectos:

- El riesgo de contagio en inmunosuprimidos por cáncer es mayor (6).

- La mortalidad en pacientes oncológicos contagiados por SARS-CoV-2 es mayor (6).

- Se necesita contar con suficientes camas de hospitalización para cubrir los ingresos de pacientes afectados por la pandemia.

Es fundamental establecer medidas en los servicios quirúrgicos para proteger al paciente y al equipo de salud, para lo cual se proponen, de forma general, estas estrategias (11):

- Fortalecer la toma de decisiones en juntas virtuales multidisciplinarias para los pacientes que necesiten biopsias percutáneas, EBUS, broncoscopia o cirugía, dado que se requiere evaluar aquellas condiciones especiales durante la pandemia que modifican el actuar usual, e identificar acciones que son requeridas para mitigar el riesgo de contagio, teniendo en cuenta las limitaciones y restricciones de los procedimientos endoscópicos, así como la limitación de recursos disponibles durante la pandemia.

- Posponer la terapia adyuvante, de común acuerdo con el paciente, explicando los riesgos y beneficios, teniendo en cuenta el riesgo de progresión de la enfermedad, o considerar ofrecer esquemas de tratamiento con menor toxicidad y soportados con factores estimulantes de colonias.

- Establecer, según la fase de la pandemia, la realización de procedimientos quirúrgicos que ofrezcan la menor morbilidad y recuperación más rápida.

- Proveer los EPP para pacientes con cáncer o sobrevivientes de cáncer, de acuerdo con las rutas propias de cada institución.
- En pacientes con cáncer infectados con COVID-19 es necesario una vigilancia y tratamiento más intensivos, especialmente en los mayores con comorbilidades.

- Aprovechar al máximo las opciones diagnósticas menos invasivas, cuyo rendimiento sea apoyado en la literatura y apropiado para el caso.

- Establecer canales de comunicación efectivos para el seguimiento de los pacientes que están en un proceso diagnóstico o terapéutico, para informarles a tiempo el momento adecuado para acudir al hospital.

- Se debe evitar, en lo posible, la hospitalización prolongada.

La pandemia por COVID-19, como emergencia de salud pública, en el ámbito quirúrgico implica:

- Prevenir el contagio de pacientes, familiares y personal de salud.

- Realizar la priorización de pacientes que requieran diagnóstico histológico, estadificación invasiva o tratamiento quirúrgico, según cada fase de la pandemia.

- Optimizar las camas de hospitalización para permitir el ingreso a pacientes infectados por SARSCoV-2. Dado el mayor riesgo de complicaciones para los pacientes con cáncer, se deben establecer rutas de atención separadas e idealmente áreas de cirugía y hospitalización diferentes a las de la población general.

- Integrar el sistema de salud para atender estos casos en los centros de referencia apropiados y con menor afluencia de casos de COVID-19.

El tiempo para definir la cirugía dependerá del momento de evolución de la pandemia, del estadio de la enfermedad y de las condiciones del enfermo, por lo que se sugiere priorizar inicialmente, mientras exista adecuada disponibilidad de recursos, a los pacientes susceptibles de curación quirúrgica o con necesidad prioritaria de diagnóstico histológico de la siguiente manera:

- Si hay fuerte sospecha de cáncer pulmonar por los hallazgos de un examen de rutina o un PET-CT o la presencia de una lesión maligna confirmada.

Revista Colombiana de Neumología Vol. 32 N. ${ }^{\circ} 1$ | 2020 
- Si existen limitaciones técnicas para el diagnóstico histológico de un tumor central o compromiso mediastinal, se debe revisar el caso en junta multidisciplinaria y considerar ofrecer tratamiento neoadyuvante previa explicación de la situación y riesgo $\mathrm{y}$ beneficio de la conducta, y según los factores de riesgo de la neoplasia y la comorbilidad del paciente.

- Para iniciar la quimioterapia neoadyuvante en pacientes que no puedan ser llevados a cirugía curativa por saturación del sistema de salud, se debe contar siempre con un diagnóstico histológico (12).

El American College of Surgeons divide la pandemia en tres fases (13): en la primera, aún se cuenta con ventiladores, los recursos hospitalarios aún no se han agotado y se restringe la cirugía para los casos en que la sobrevida pueda verse comprometida en los próximos meses. Se sugiere operar tumores pulmonares sólidos o predominantemente sólidos ( $>50 \%)$, mayores de $2 \mathrm{~cm}$, estadio ganglionar N0 o tumores pulmonares que requieran estadificación mediastinal o pleural para empezar tratamiento. Se aconseja diferir tumores menores de $2 \mathrm{~cm}$ o aquellos con predominio en vidrio esmerilado. En el escenario urgente, fase II, solo deberían operarse pacientes con amenaza para la vida si no se operan en los próximos días, es decir, con infecciones u otras complicaciones, como sangrado, empiema y hemotórax. En la fase III se trata de compromiso vital en horas, en cáncer de pulmón habría contadas indicaciones, como hemoptisis masivas o tumores asociados con sepsis. Esta es una perspectiva útil y clara.

En China, en lo que ellos llamaron el período más inestable de la pandemia, decidieron dividir los pacientes quirúrgicos de tórax en dos categorías, estas son (12):

- Cirugía optativa: definida como la cirugía que se puede aplazar bajo vigilancia activa por más de 3 meses, que incluye opacidades múltiples en vidrio esmerilado, nódulos pulmonares con un diámetro $<3 \mathrm{~cm}$, especialmente si sus características sugieren $70 \%$ de posibilidad de benignidad, y enfermedades pulmonares benignas que pueden tratarse de forma conservadora. Para este grupo se recomienda no realizar cirugía durante la pandemia.
- Cirugía de término limitado: definida como la mejor operación dentro de 1 mes. Se incluyen los pacientes con cáncer de pulmón claramente diagnosticado o con fuerte sospecha clínica, con un diámetro $>3 \mathrm{~cm}$, con indicación quirúrgica (estadios I y II); dicha cirugía debe realizarse previa exclusión de neumonía por SARS-CoV-2. Según la experiencia en China se propone tratamiento quirúrgico, si las condiciones locales y el estado de la pandemia lo permiten.

\section{Recomendaciones para el procedimiento}

Con el fin de reevaluar la seguridad de la cirugía en relación con infección por SARS-CoV-2, el día de la consulta preanestésica se debe realizar un cuestionario sobre los siguientes aspectos:

- ¿Ha presentado tos seca como síntoma de reciente aparición?

- ¿Ha presentado fiebre?

- ¿Ha viajado en los últimos 14 días a zonas de posible contagio de COVID-19?

- ¿Ha tenido contacto con pacientes sospechosos o confirmados para SARS-CoV-2 en los últimos 14 días?

El día de la cirugía debe aplicarse nuevamente el cuestionario anterior, el paciente debe acudir al hospital con mascarilla quirúrgica convencional y se debe realizar medición de temperatura. La recomendación final es ponderar cada caso de acuerdo con las recomendaciones anteriormente enunciadas, las del propio paciente, la situación institucional de disponibilidad de recursos y el número de casos locales confirmados para COVID-19; todo en estrecha comunicación entre el personal asistencial y administrativo de la institución.

\section{Recomendaciones para el tratamiento del cáncer durante la pandemia por COVID-19}

Los pacientes con diagnóstico de cáncer de pulmón se deben clasificar en tres escenarios terapéuticos: el paciente con diagnóstico nuevamente, en espera de tratamiento quirúrgico curativo o quimioterapia adyuvan- 
te; el paciente en tratamiento sistémico con intención curativa; y el paciente candidato a tratamiento sistémico con intención paliativa.

En las circunstancias actuales es fundamental, antes de decidir si se debe administrar o no un tratamiento oncológico sistémico, considerar el beneficio frente al riesgo asociado con la posibilidad de contagio por el SARS-CoV-2, y sus potenciales consecuencias, especialmente la necesidad de requerir ingreso hospitalario por complicaciones graves.

En pacientes en tratamiento dentro de ensayos clínicos se deben seguir estrictamente las recomendaciones del protocolo, utilizando las posibilidades de retrasar o ajustar las dosis que ofrece el mismo, para adaptarse a la situación asistencial y epidemiológica. El investigador siempre debe tomar las decisiones más adecuadas para la salud y seguridad del paciente.

En todo paciente que reciba tratamiento oncológico sistémico durante la pandemia de la infección por el SARS-CoV-2 se debe incluir en el consentimiento informado la posibilidad de aumento del riesgo de contagio y de efectos secundarios y complicaciones relacionados con la infección.

En caso de necesidad de cirugía oncológica con intención curativa se debe evaluar cada caso, y determinar cada uno de forma individual, con base en los daños potenciales de retrasar la cirugía relacionada con el cáncer a tratar.

\section{Paciente con diagnóstico de cáncer pulmonar nuevamente en espera de inicio de tratamiento quirúrgico curativo o quimioterapia adyuvante}

Espera de tratamiento: deben evaluarse cuidadosamente de acuerdo con el estadio clínico, la carga tumoral y el estado general del paciente.

Los pacientes en estadios I y II, candidatos a cirugía como tratamiento inicial, deben ser llevados a cirugía idealmente en cuanto se completa el diagnóstico y la estadificación, sin embargo, en la emergencia sanitaria por la pandemia podrían diferirse hasta 3 meses, siempre y cuando no se comprometa la supervivencia del paciente, lo cual es factible en la mayoría de los cáncer de pulmón de célula no pequeña (CPNCP) estadio I, especialmente en T1a y T1b $(<2 \mathrm{~cm})$, sobre todo si estamos ante nódulos en vidrio esmerilado. Como en cualquier otra circunstancia, si se sospecha de infección por coronavirus o por otro patógeno, la cirugía deberá retrasarse hasta evidenciar resolución completa del cuadro respiratorio y RT-PCR para SARS-CoV-2 negativa.

Para los pacientes que esperan quimioterapia adyuvante después de la cirugía, las decisiones deben tomarse en función del diagnóstico y el estadio patológico, del estado general del paciente, de los factores de riesgo y de los indicadores pronósticos. Para los pacientes con cáncer de pulmón posoperatorio con estadio clínico más temprano y mejor pronóstico, el tiempo para comenzar la quimioterapia adyuvante puede extenderse, sin detrimento de la supervivencia de los enfermos. Los estudios han demostrado que la quimioterapia que se inició entre 57 y 127 días después de la cirugía no aumentó la mortalidad (HR 1,037) (14). Se ha visto que los pacientes con recuperación lenta después de la cirugía de cáncer de pulmón pueden aún beneficiarse de la quimioterapia adyuvante tardía cuando se inicia 4 meses después de la cirugía (14).

Siempre debe tenerse en cuenta:

- No iniciar tratamientos de quimioterapia potencialmente inmunosupresora en pacientes con sospecha de infección por SARS-CoV-2 o historia de contacto de riesgo.

- No iniciar tratamientos de quimioterapia potencialmente inmunosupresora en pacientes en los que la demora de 2-3 semanas no suponga un riesgo en la vida, en pacientes asintomáticos con contacto reciente $(\leq 14$ días) positivo o sospechoso para SARS-CoV-2.

\section{Paciente con diagnóstico de cáncer pulmonar en tratamiento, con intención curativa}

- A todo paciente que ingrese a los servicios de oncología para recibir tratamiento sistémico se le debe ofrecer todas las medidas de protección establecidas, realizar toma de temperatura (idealmente oral), en caso de ser superior de $37,5^{\circ} \mathrm{C} \mathrm{y}$ 
de presentar síntomas respiratorios se debe iniciar un protocolo de diagnóstico para SARS-CoV-2, según cada institución.

- Generar el proceso de toma de muestras para SARS-CoV-2 en el mismo instituto de oncología, y definir la administración del tratamiento de acuerdo con los resultados.

- Optimizar los tiempos de atención en las salas de quimioterapia, considerar establecer más turnos para que los pacientes estén separados por 2 metros durante la infusión.

- Considerar el soporte con factores estimulantes de colonias.

- Aplazar la administración de fármacos que pueden ser diferibles, como bifosfonatos y denosumab.

- Priorizar los pacientes que requieran terapia sistémica, teniendo en cuenta el nivel de inmunosupresión asociado con el tratamiento o con las condiciones propias del paciente, la capacidad de respuesta con los recursos disponibles, el balance riesgo/beneficio respecto a la posibilidad de adquirir la infección por SARS-CoV-2, el tratamiento no óptimo y la posibilidad de conseguir los beneficios esperados (4).

- Implementar esquemas de tratamiento oncológico sistémico que permitan al paciente realizar el menor número de visitas al centro oncológico/hospital ajustados a las características de la neoplasia, a los factores de riesgo y a la comorbilidad de cada paciente.

- Considerar el cambio de terapia, teniendo en cuenta la dosificación de la frecuencia de uso, el tiempo de tratamiento actual, el faltante y el total para terminar la terapia, así como las facilidades del paciente para asistir a los tratamiento, y factores propios de la logística en el centro oncológico y del momento o fase de la pandemia que se viva en el país (4).

- Realizar teleconsulta de seguimiento de tratamiento.

- En los casos en los que se requiera la administración de quimio/radioterapia, considerar realizar este tratamiento en forma secuencial y no concomitante.
- Evaluar al paciente con cáncer pulmonar y su entorno, ofreciéndole una atención integral y humana en momentos de grandes dificultades $(15,16)$.

- Diferir las consultas de los supervivientes sanos que no reciban tratamiento oncológico activo. Las consultas de soporte, como nutrición o psicología, también pueden realizarse por teleconsulta (9).

\section{Paciente con diagnóstico de cáncer pulmonar en tratamiento sistémico, con intención paliativa}

Para los pacientes en tratamiento sistémico con intención paliativa se debe considerar:

- Todos los pacientes candidatos a tratamiento sistémico deben tener los resultados del perfil molecular de la neoplasia para decidir el tratamiento oncológico basado en dicho resultado.

- Considerar cambios en los esquemas de tratamiento sistémico, que permitan al paciente realizar el menor número de visitas al centro oncológico/hospital, ajustados a las características de la neoplasia, respuesta tumoral, factores de riesgo y comorbilidad de cada paciente.

- Realizar teleconsulta de seguimiento de tratamiento.

- Optimizar la terapia paliativa en casa, asegurando la disponibilidad de los medicamentos y elementos de manejo de dolor y disnea.

- Educar en medidas de autocuidado, utilizando los métodos de telemedicina, en caso de requerirlo.

\section{Recomendaciones de manejo de la radioterapia}

El uso de la radioterapia en el cáncer de pulmón desempeña un papel determinante y potencialmente curativo en el tratamiento de pacientes en estados iniciales, localmente avanzados y metastásicos. Durante la actual pandemia se ha determinado mantener la continuidad del manejo con radiación ionizante, teniendo en cuenta el acrónimo RADS (17):

- Remote visits (estimular las teleconsultas y teleseguimiento). 
- Avoid radiation (evitar la radioterapia).

- Defer radiation (retrasar, cuando sea posible y aceptable, la administración de la radioterapia).

- Shorten radiation (acortar la duración de los tratamientos, y favorecer el empleo de esquemas cortos y ultracortos de irradiación).

Las guías ampliamente difundidas para el manejo de pacientes oncológicos durante la actual pandemia, siempre han recomendado la disminución de riesgos tanto para grupos tratantes, como para pacientes (17-19). Una de las formas de cumplir con este objetivo es la reducción de desplazamientos y visitas a las unidades de tratamiento de radioterapia, mediante el uso de esquemas hipofraccionados, caracterizados por un menor número de sesiones de tratamiento, siempre que sea posible $(19,20)$.

En estadios iniciales T1-2 N0 está completamente indicado el uso de la radiocirugía corporal (Stereotatic Body Radiation Therapy [SBRT]) para lesiones centrales y periféricas, en esquemas que van de 1 a 8 fracciones, dependiendo de la distancia que existe desde la pared torácica $u$ otros órganos sensibles, al tamaño de la lesión, así como la dosimetría obtenida en cada planeación de tratamiento $(17,21)$.

Adicional al uso de SBRT, la radioterapia de intensidad modulada (IMRT) se sugiere como técnica para realizar tratamientos hipofraccionados, pues es así como se logra una disminución adecuada de las dosis de radiación a los órganos sanos adyacentes, respetando las dosis de tolerancia establecidas para cada uno de ellos, y disminuyendo la toxicidad aguda y tardía $(17,18,22)$.

Para estadios localmente avanzados, el tratamiento estándar es de esquemas concomitantes con quimioterapia, seguidos de durvalumab. Sin embargo, de acuerdo con la evolución de la pandemia, lo más sensato es hacer esquemas secuenciales para disminuir el riesgo de toxicidad grave asociada, comenzando por el tratamiento disponible, idealmente quimioterapia de inducción. En casos sintomáticos, como sangrado u obstrucción, la radioterapia sería la opción primaria.

En caso de adyuvancia hay que valorar retrasar el inicio de la radioterapia por 4 a 6 semanas, dependien- do de la fase de la pandemia (19). Para paliación se utilizará hipofraccionamiento extremo.

Las técnicas de radioterapia consideran la radioterapia guiada con imágenes (IGRT), preferiblemente hipofraccionada (15-20 fracciones), sin incluir las áreas ganglionares electivas o la realización de boost integrado simultáneo (SIB), según la experticia del centro (21). No se recomienda el hipofraccionamiento en pacientes con cáncer de pulmón de célula no pequeña en el escenario adyuvante posoperatorio, ni tampoco en pacientes con cáncer de pulmón de célula pequeña que requieran profilaxis del sistema nervioso central (21). Quien tenga la posibilidad de realizar cone-beam CT diario, puede alertar ante cualquier cambio sugestivo de neumonía vírica durante el tratamiento (17).

En caso de pacientes con SARS-CoV-2 positivo, sin síntomas graves, cuando sea mandatoria la radioterapia prioritaria o urgente, se atenderán en la unidad de radioterapia al final del turno en el acelerador lineal, con todo el protocolo de protección personal, así como de desinfección. Si el paciente no es urgente se esperará la resolución de la infección con prueba negativa.

En cada institución es fundamental mejorar la comunicación entre todos los actores, para decidir en junta multidisciplinaria la opción para cada paciente, así como tener comunicación fluida, en lo posible, con el grupo tratante de COVID-19, y permitir diferenciar los hallazgos pulmonares de un paciente con cáncer de pulmón.

\section{Recomendaciones para las instituciones}

- Reorientar la capacidad instalada de los centros oncológicos, según la fase de la pandemia y los requerimientos regionales.

- Iniciar y mantener un registro poblacional propio de pacientes con cáncer y COVID-19.

- Brindar servicios de terminalidad en casa.

- El personal del hospital que haya tenido contacto con un caso confirmado debe realizarse la prueba para infección por SARS-CoV-2 y aislarse hasta confirmar la ausencia de infección. 
- Cualquier médico o paramédico que presente síntomas respiratorios significativos debe comunicárselo de forma urgente telefónicamente a su supervisor. Abstenerse de acudir al puesto de trabajo, realizarse la prueba para infección SARS-CoV-2 y mantener aislamiento hasta confirmación de ausencia de infección.

\section{Recomendaciones para los pacientes con diagnóstico de cáncer y sus cuidadores durante la pandemia por COVID-19}

Los pacientes con cáncer deben ser instruidos, a través de las diferentes tecnologías disponibles, en acciones de autocuidado y disminución de riesgo de contagio. Anotamos aquí las medidas de prevención recomendadas por nuestro grupo, apoyándonos en recomendaciones internacionales de países que ya han sufrido de manera amplia la epidemia $(5,23)$.

- Acate las recomendaciones de aislamiento suministradas por el gobierno nacional.

- No ir a hospitales, a no ser que sea absolutamente necesario.

- Haga uso de los servicios de medicina a distancia disponibles en su institución médica.

- En caso de requerir asistir a hospitales, citas médicas o estudios de diagnóstico, realice lavado de manos o higienización con alcohol glicerinado frecuentemente, y use mascarilla.

- Cuando salga debe ir con máximo 1 acompañante, quien debe tener las mismas precauciones que usted.

- Vigile su temperatura. En caso de fiebre (temperatura superior o igual de $38,3{ }^{\circ} \mathrm{C}$ ) informe virtualmente a su médico. Si dispone de un servicio de medicina domiciliaria, informe telefónicamente sus síntomas para evaluar la opción a seguir.

- En caso de la aparición de síntomas sugestivos de COVID-19, como dolor de garganta, fiebre o tos, informe a su médico y siga las recomendaciones locales para casos de COVID-19.

- En caso de tener síntomas compatibles con COVID-19 y sensación de ahogo, alerte a las autoridades locales a través de la línea telefónica indicada, para ser trasladado a un centro asistencial. Según cambien las recomendaciones locales, utilice aquellas que estén vigentes.

- Mantenga una adecuada higiene personal y de su hogar; recuerde limpiar aquellos lugares frecuentemente usados, como perillas de las puertas, interruptores de energía, mesones, grifos, baños, entre otros.

- Mantenga una alimentación adecuada para sus enfermedades, según las indicaciones dadas por su nutricionista.

- Respete sus horarios de sueño y descanso.

- Mantenga el buen ánimo. Cuidar de su estado emocional le permite estar enfocado en las cosas realmente importantes durante la contingencia de la pandemia.

\section{Retos en el cuidado del paciente con cáncer pulmonar durante la pandemia por COVID-19 en Colombia}

Es necesario utilizar las tecnologías de evaluación a distancia disponibles. Si un profesional de la salud requiere medidas de autoaislamiento, aun así puede ayudar a través de actividades de consulta, reuniones de equipo, toma de decisiones, análisis de datos y tareas que sean posible realizar de forma remota y teledirigidas (4).

Es importante ofrecer apoyo y soporte al equipo de trabajo para el bienestar mental durante la pandemia. Una vez se logre sobrepasar las dificultades propias de la pandemia, es importante reevaluar los casos individualmente, en busca de ajustar a la mejor terapia disponible para cada caso.

\section{Referencias}

1. World Health Organization [Internet]. Cancer. Última visita: 22 de marzo de 2020. Disponible en: https://www.who.int/ westernpacific/health-topics/cancer

2. World Health Organization [Internet]. Cancer today. Última visita: 22 de marzo de 2020. Disponible en: http://gco.iarc. fr/today/home

3. Huang C, Wang Y, Li X, Ren L, Zhao J, Hu Y, et al. Clinical features of patients infected with 2019 novel coronavirus in Wuhan, China. Lancet. 2020;395(10223):497-506. doi: 10.1016/S0140-6736(20)30183-5. 
4. National Institute for Health and Care Excellence [Internet]. COVID-19 rapid guideline: delivery of systemic anticancer treatments. Última visita: 23 de marzo de 2020. D i s ponible en: https://www.nice.org.uk/guidance/ng161

5. Zhao Z, Bai H, Duan J, Wang J. Recommendations of individualized medical treatment and common adverse events management for lung cancer patients during the outbreak of COVID-19 epidemic. Thorac Cancer. 2020 Jun;11(6):1752-1757.

6. Liang W, Guan W, Chen R, Wang W, Li J, Xu K, et al. Cancer patients in SARS-CoV-2 infection: a nationwide analysis in China. Lancet Oncol. 2020;21(3):P335-7. doi: https://doi. org/10.1016/S1470-2045(20)30096-6.

7. Journal of the National Comprehensive Cancer Network [Internet]. JNCCN: How to manage cancer care during COVID-19 pandemic. Última visita: 22 de marzo de 2020. Disponible en: https://www.nccn.org/about/news/newsinfo. aspx?NewsID $=1949$

8. $\mathrm{Xu} \mathrm{Y,} \mathrm{Liu} \mathrm{H,} \mathrm{Hu} \mathrm{K,} \mathrm{Wang} \mathrm{M.} \mathrm{[Clinical} \mathrm{management} \mathrm{of}$ lung cancer patients during the outbreak of 2019 novel coronavirus disease (COVID-19)]. Zhongguo Fei Ai Za Zhi. 2020;23(3):136-41. doi: 10.3779/j.issn.10093419.2020.03.02.

9. Dalton CB, Corbett SJ, Katelaris AL. Pre-emptive low cost social distancing and enhanced hygiene implemented before local COVID-19 transmission could decrease the number and severity of cases. Med J Aust. 2020;212(10):1.

10. European Society for Medical Oncology [Internet]. ESMO management and treatment adapted recommendations in the COVID-19 era: lung cancer. Última visita: 11 de abril de 2020. Disponible en: https://www.esmo.org/guidelines/ cancer-patient-management-during-the-covid-19-pandemic/ lung-cancer-in-the-covid-19-era

11. Wong J, Goh QY, Tan Z, Lie SA, Tay YC, Ng SY, et al. Preparing for a COVID-19 pandemic: a review of operating room outbreak response measures in a large tertiary hospital in Singapore. Can J Anesth Can Anesth [Internet]. 2020;67:732-45. doi: https://doi.org/10.1007/s12630-020-01620-9.

12. Li X, Liu M, Zhao Q, Liu R, Zhang H, Dong M. [Preliminary recommendations for lung surgery during 2019 novel coronavirus disease (COVID-19) epidemic period. Zhongguo Fei Ai Za Zhi. 2020;23(3):133-5.

13. Nelson R. Guidelines on delaying cancer surgery during COVID-19. Medscape [Internet]. Última visita: 14 de abril de 2020. Disponible en: http://www.medscape.com/viewarticle/927568
14. Salazar MC, Rosen JE, Wang Z, Arnold BN, Thomas DC, Herbst RS, et al. Association of delayed adjuvant chemotherapy with survival after lung cancer surgery. JAMA Oncol. 2017;3(5):610-9. doi: 10.1001/jamaoncol.2016.5829.

15. Thompson AK, Faith K, Gibson JL, Upshur RE. Pandemic influenza preparedness: an ethical framework to guide decisionmaking. BMC Med Ethics. 2006;7(1):12. doi: 10.1186/14726939-7-12.

16. Institut Borja de Bioètica [Internet]. Recursos éticos sobre el coronavirus (COVID-19). Última visita: 23 de marzo de 2020. Disponible en: https://www.iborjabioetica.url.edu/es/comunicacion/recursos-eticos-sobre-el-coronavirus-covid-19

17. Guckenberger M, Belka C, Bezjak A, Bradley J, Daly ME, DeRuysscher D, et al. Practice recommendations for lung cancer radiotherapy during the COVID-19 pandemic: an ESTRO-ASTRO consensus statement. Radiother Oncol. 2020;146:223-9. doi: https://doi.org/10.1016/j.radonc.2020.04.001.

18. Wu AJ, Rimner A, Shepherd AF, Yorke E, Simone CB, Gomez DR, et al. Thoracic radiation therapy during coronavirus disease 2019: provisional guidelines from a comprehensive cancer center within a pandemic epicenter. 2020;5(4):603-7. doi: https://doi.org/10.1016/j.adro.2020.04.008.

19. Faivre-Finn C, Fenwick JD, Franks KN, Harrow S, Hatton $\mathrm{MQF}$, Hiley $\mathrm{C}$, et al. Reduced fractionation in lung cancer patients treated with curative-intent radiotherapy during the COVID-19 pandemic. Clin Oncol. 2020;32(8):481-9. doi: https:// doi.org/10.1016/j.clon.2020.05.001.

20. van Doremalen N, Bushmaker T, Morris DH, Holbrook MG, Gamble A, Williamson BN, et al. Aerosol and surface stability of SARS- CoV-2 as compared with SARS-CoV-1. N Engl J Med. 2020;382:1564-7. doi: 10.1056/NEJMc2004973.

21. Swanick CW, Lin SH, Sutton J, Naik NS, Allen PK, Levy $\mathrm{LB}$, et al. Use of simultaneous radiation boost achieves high control rates in patients with none small-cell lung cancer who are not candidates for surgery or conventional chemoradiation. Clin Lung Cancer. 2015;16(2):156-63. doi: 10.1016/j. cllc.2014.10.005.

22. Kumar S, Chmura S, Robinson C, Lin SH, Gadgeel SM, Donington J. Alternative multidisciplinary management options for locally advanced non-small cell lung cancer during the COVID-19 global pandemic. J Thorac Oncol. 2020;15(7):113746. doi: https://doi.org/10.1016/j.jtho.2020.04.016.

23. Pino L, Pérez C, Cardona A, Triana I. Cancer center recommendation to mitigate COVID-19 impact in patients with cancer: low resource settings version. Carta al editor. JCO Global Oncology. 2020;6:569-70. doi: 10.1200/GO.20.00093. 